\title{
Time Dependence of Intra-fractional Motion in Spinal Stereotactic Body Radiotherapy
}

\author{
RYUTA HIRAI $^{1}$, YU OHKUBO ${ }^{2}$, MITSUNOBU IGARI ${ }^{1}$, YU KUMAZAKI ${ }^{1}$, TOMOMI AOSHIKA ${ }^{1}$, \\ YASUHIRO RYUNO ${ }^{1}$, SATOSHI SAITO ${ }^{1}$, TAKANORI ABE ${ }^{1}$, SHIN-EI NODA ${ }^{1}$ and SHINGO KATO ${ }^{1}$ \\ ${ }^{1}$ Department of Radiation Oncology, Saitama Medical University International Medical Center, Saitama, Japan; \\ ${ }^{2}$ Department of Radiation Oncology, Saku Central Hospital Advanced Care Center, Nagano, Japan
}

\begin{abstract}
Background/Aim: Positional uncertainty in spinal stereotactic body radiotherapy (SBRT) may cause fatal error, therefore, we investigated the intra-fractional spinal motion during SBRT and its time dependency. Patients and Methods: Thirty-one patients who received SBRT using CyberKnife were enrolled in the study. $2 \mathrm{D} \mathrm{kV}$ $X$-ray spine images in two directions were taken before and during treatment. Image acquisition intervals during treatment were set at 35-60 sec. Automatic image matchings were performed between the reference digital reconstructed radiography $(D R R)$ and live images, and the spinal position displacements were logged in six translational and rotational directions. If the displacements exceeded $2 \mathrm{~mm}$ or 1 degree, the treatment beam delivery was interrupted and the patient position was corrected by moving couch, and the couch adjustments were also logged. Based on the information, the time-dependent accumulated translational and rotational displacements without any couch adjustments were calculated. Results: Spinal position displacements in all translational and rotational directions were correlated with elapsed treatment time. Especially, Right-Left displacements of $>1 \mathrm{~mm}$ and $>2 \mathrm{~mm}$ were observed at 4-6 and 8-10 min after treatment initiation, respectively. Rotational displacements in the Yaw direction $>1^{\circ}$ were observed at 10-15 min after treatment initiation. Conclusion: The translational and rotational displacements systematically increased with elapsed treatment time. It is suggested that the spine position should be checked at least
\end{abstract}

This article is freely accessible online.

Correspondence to: Ryuta Hirai, Department of Radiation Oncology, Saitama Medical University International Medical Center, 1397-1 Yamane, Hidaka, Saitama 350-1298, Japan. Tel and Fax: +81 429844136, e-mail: rhirai@ saitama-med.ac.jp

Key Words: Stereotactic body radiotherapy, spinal metastasis, intrafractional motion, setup margin. every 4-6 min or the treatment time should be limited within 4-6 minutes to ensure the irradiation accuracy within the millimeter or submillimeter range.

Developments in chemotherapy, molecular targeted therapy, and immunotherapy [e.g., programmed death-1 (PD1)/Programmed death-ligand 1 (PD-L1) inhibitors] have contributed to the prolonged survival of patients with distant metastatic disease. Maintaining the quality of life (QOL) of patients by controlling distant metastatic lesions is an important challenge to address. The spine is one of the most common sites of metastasis, causing pain and negatively impacting QOL. Spinal metastases have been treated using palliative radiotherapy with a dose of 8 Gy in a single fraction or 20-30 Gy in 5-10 fractions using conventional two-dimensional radiotherapy (2DRT) or three-dimensional conformal radiotherapy (3DCRT). Stereotactic body radiotherapy (SBRT) is increasingly being used for oligometastases of the spine. SBRT enables the delivery of high radiation doses to the tumor specifically while minimizing the dose to the surrounding normal tissues, such as the spinal cord, which may provide longer pain relief and better tumor control when compared with conventional radiotherapy $(1,2)$. Moreover, some studies have reported the effectiveness of re-irradiation using SBRT for recurrent spinal metastases after conventional radiotherapy (3).

Intra-fractional motion errors are critical in SBRT for spinal metastases. Therefore, various types of image-guided radiotherapy (IGRT) are performed in parallel to minimize the displacement of a patient's position during treatment (4, 5 ). Even so, it is difficult to perform real-time correction of a patient's position during treatment in several treatment devices. The treatment duration of SBRT varies by i) the treatment device, ii) the site of irradiation, and iii) the prescribed dose, but is generally longer than that of a conventional 3DCRT. Regarding the treatment device, several studies on the treatment planning have reported the treatment time of approximately 10 min using TrueBeam (Varian Medical systems, Palo Alto, CA, USA) and 20-30 
Table I. Patient characteristics.

\begin{tabular}{|c|c|c|}
\hline & Characteristic & $\begin{array}{l}\text { Number } \\
\text { of cases }\end{array}$ \\
\hline \multirow{5}{*}{$\begin{array}{l}\text { Tumor } \\
\text { location }\end{array}$} & Lung (primary or metastatic) & 22 \\
\hline & $\begin{array}{l}\text { Rectum (postoperative pelvic } \\
\text { recurrence) }\end{array}$ & 1 \\
\hline & $\begin{array}{l}\text { Prostate (postoperative pelvic } \\
\text { recurrence) }\end{array}$ & 1 \\
\hline & Spinal body (thoracic) & 2 \\
\hline & $\begin{array}{l}\text { Lymph node metastasis } \\
\text { (neck/abdomen/pelvis) }\end{array}$ & 6 \\
\hline \multirow{3}{*}{$\begin{array}{l}\text { Tracking } \\
\text { location }\end{array}$} & Cervical spine & 3 \\
\hline & Thoracic spine & 22 \\
\hline & Lumbar spine & 7 \\
\hline Prescribed dose & 12-60 Gy & - \\
\hline Fractions & 3-8 fractions & - \\
\hline $\begin{array}{l}\text { Mean image } \\
\text { interval }\end{array}$ & $1 \min 9 \sec \pm 11 \mathrm{sec}$ & - \\
\hline $\begin{array}{l}\text { Mean treatment } \\
\text { time }\end{array}$ & $34 \min 12 \mathrm{sec} \pm 10 \min 34 \mathrm{sec}$ & - \\
\hline
\end{tabular}

min using TomoTherapy (Accuray, Inc., Sunnyvale, CA, USA) $(6,7)$. With an increased treatment duration, intrafractional motion error may occur more frequently during SBRT. Therefore, the time dependent intra-fractional motion of the spine should be accurately assessed.

CyberKnife (Accuray, Inc.) has a room-mounted X-ray imaging system for tracking the spine during treatment, and the system logs the intra-fractional data of the translational and rotational displacements of the spine. In this study, we investigated the intra-fractional spinal motion during SBRT and its time dependency by analyzing the log data.

\section{Patients and Methods}

A total of 31 patients (32 plans) who received SBRT using CyberKnife at our hospital from April 2014 to March 2020 were enrolled in this study. Patient characteristics are summarized in Table I. Patients were immobilized using a vacuum bag and a cushion placed under the knees (Figure 1). All treatments were delivered with a real-time tracking system using the spinal body near the tumor as a landmark. Log data from 32 plans conducted as initial treatment were analyzed.

Two-dimensional $\mathrm{kV} \mathrm{X}$-ray spine images in two directions were taken before and during SBRT using a room-mounted X-ray imaging system (Accuray, Inc.) associated with the CyberKnife. Image acquisition intervals during treatment were set at $35-60 \mathrm{sec}$. Since the CyberKnife system did not allow imaging during treatment beam delivery, images were acquired approximately once a minute (Table I). The timestamps of each imaging were logged. Automatic image matching using the spinal body was performed between reference digitally reconstructed radiography (DRR) images and live images using the Xsight Spine Tracking System of CyberKnife. The mismatches between DRR and live images were logged in six translational and rotational directions [Right-Left (R-L), SuperiorInferior (S-I), Anterior-Posterior (A-P), Roll, Pitch, and Yaw] (Figure
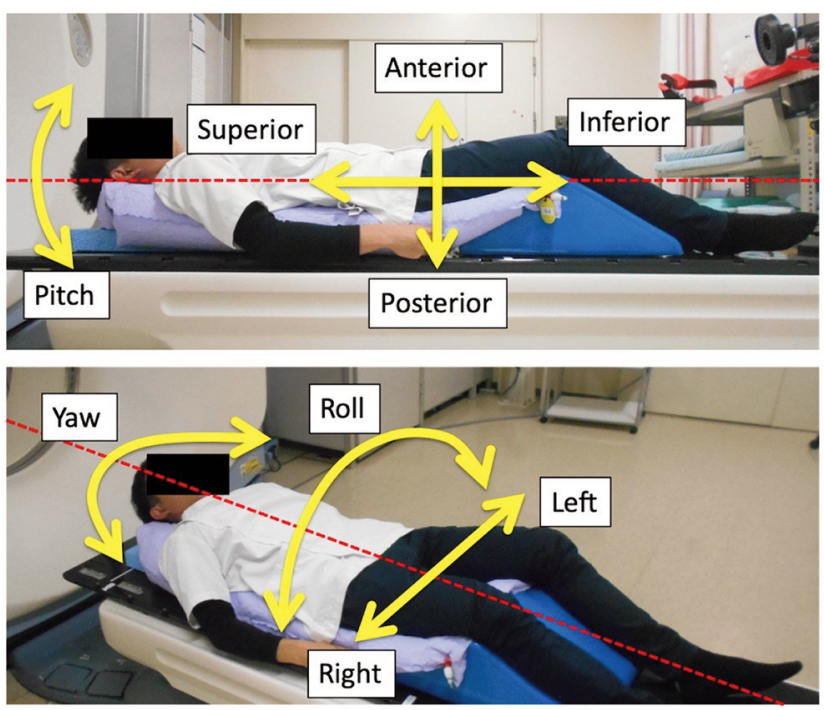

Figure 1. Patient set-up. Patients were immobilized using patientspecific vacuum bags. Yellow arrows correspond to the direction of spine translations and rotations.

1). If the displacements exceeded $2 \mathrm{~mm}$ or 1 degree, the treatment beam delivery was interrupted and the patient position was corrected by moving the couch, and the couch adjustments were also logged. Based on the information, the time-dependent accumulation of translational and rotational displacements without couch adjustments were analyzed. Positional displacements were calculated by averaging every 2 minutes for 0-10 minutes and every 5 minutes for 10-20 min. Pearson's correlation coefficients (r) were used to evaluate correlations between spinal body displacements and elapsed treatment time. Values of $p<0.05$ were considered statistically significant. This study was approved by the Institutional Review Board of our institute (Approval number: 18-106).

\section{Results}

Figures $2 \mathrm{a}$ and $\mathrm{b}$ show the time-dependent accumulation of the spinal displacements in a case with the biggest movement. Relatively large displacements in the R-L and Yaw directions were observed in this case, and the displacements tended to increase with time. Figures $2 \mathrm{c}-\mathrm{h}$ show the mean timedependent accumulation of displacements in all cases. Spinal position displacements in all translational and rotational directions were correlated with elapsed treatment time. In particular, there was a moderate correction $(r>0.4)$ between the R-L and S-I directions. Mean \pm 1 SD maximum translational displacements within $20 \mathrm{~min}$ for all patients were $1.3 \pm 0.9 \mathrm{~mm}$, $0.8 \pm 0.4 \mathrm{~mm}$, and $0.6 \pm 0.4 \mathrm{~mm}$ in the R-L, S-I, and A-P directions, respectively, and mean $\pm 1 \mathrm{SD}$ maximum rotational displacements in the Yaw, Pitch, and Roll directions were $0.6 \pm 0.3^{\circ}, 0.4 \pm 0.2^{\circ}$, and $0.5 \pm 0.3^{\circ}$, respectively.

Table II shows the proportion of cases with mean translational and rotational displacements of $>1 \mathrm{~mm},>2$ 

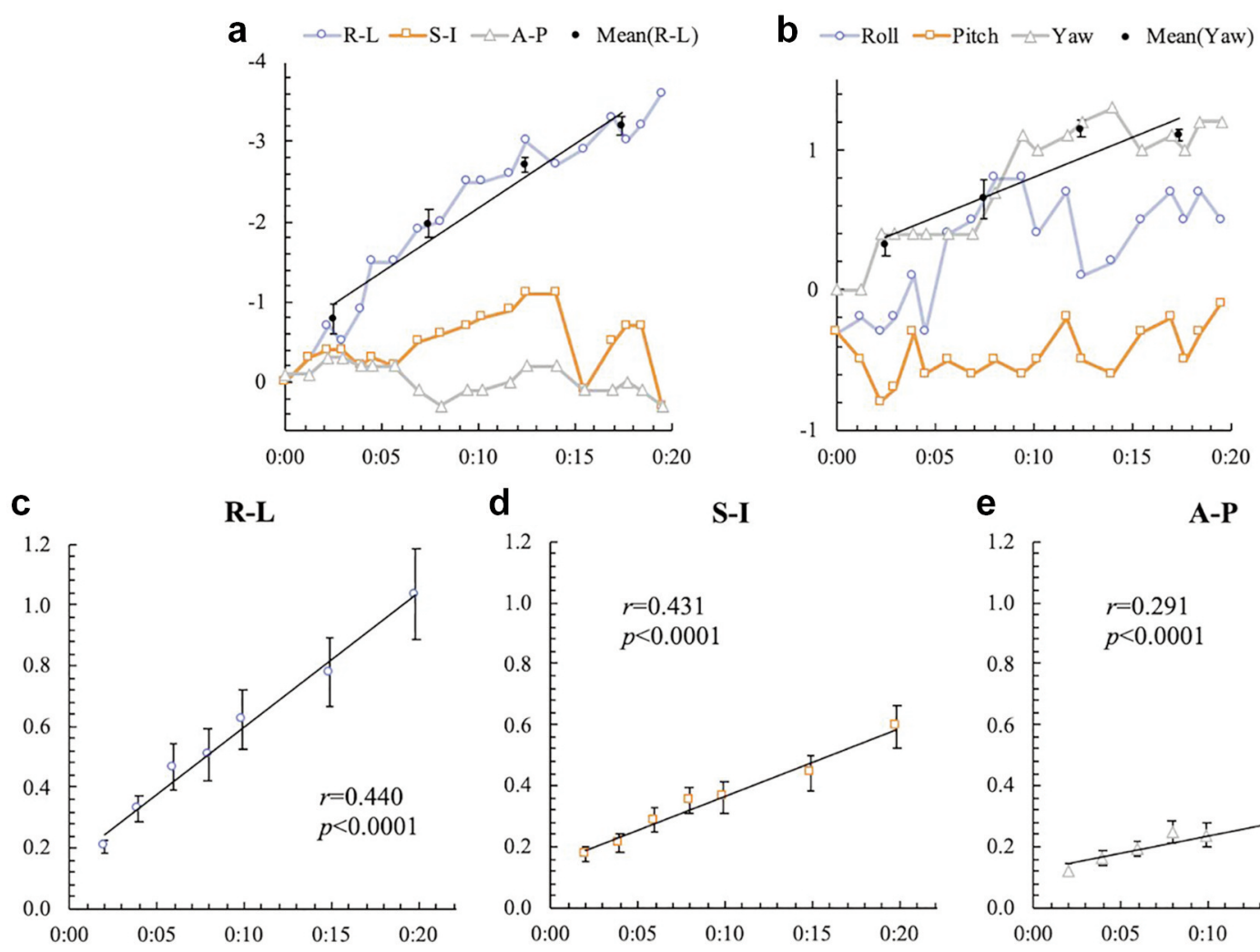

S-I
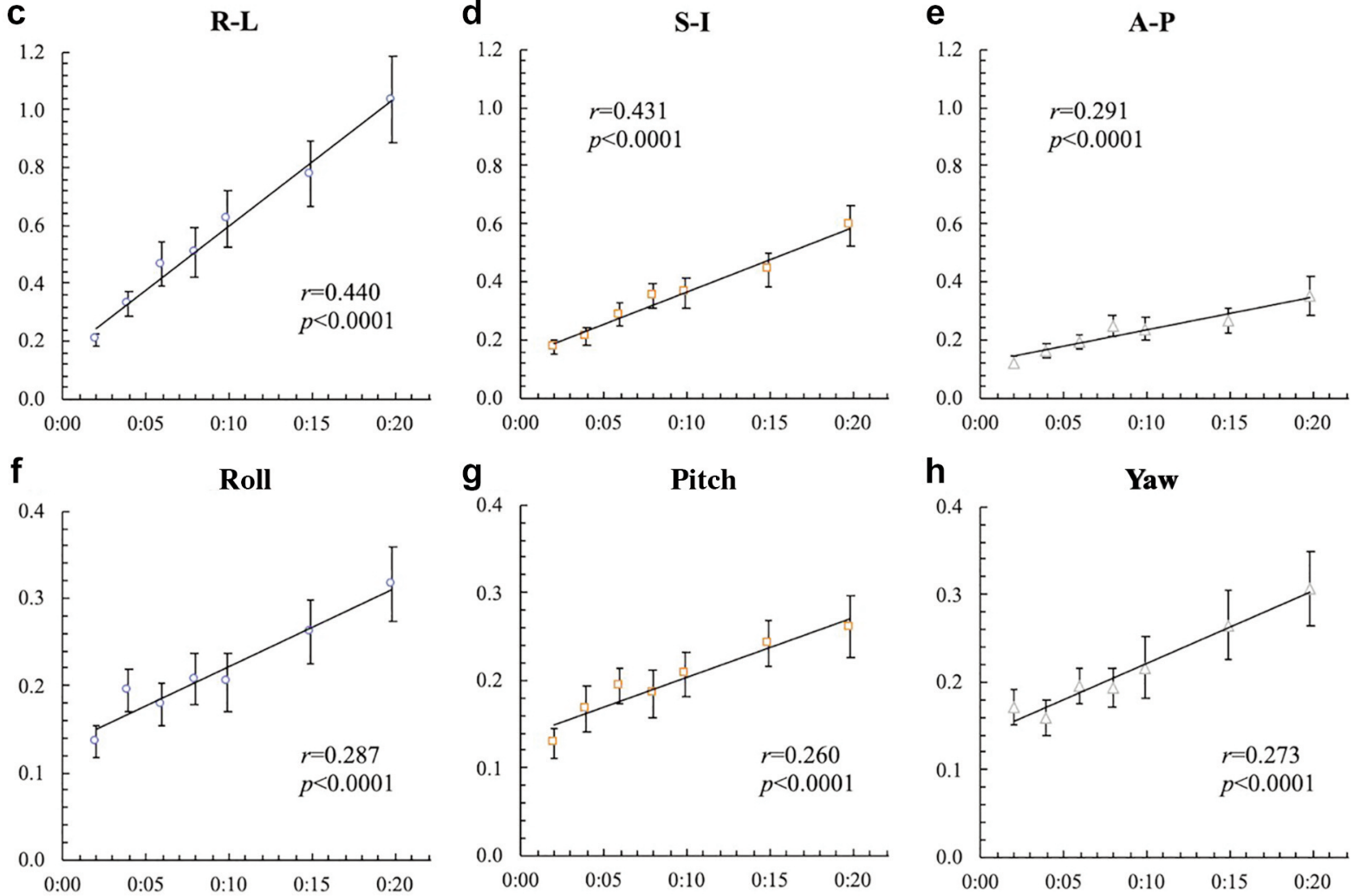

Figure 2. Time-dependent translational and rotational displacements of the spinal body. Graphs a and $b$ show the time-dependent accumulation of the spinal displacements in a case with the biggest movement. Black dots correspond to mean displacements in increments of 5 min in the RightLeft $(R-L)$ and Yaw directions, respectively. Relatively large displacements in the $R-L$ and Yaw directions were observed in this case, and the displacements tended to increase with time. Graphs $c$-h show results of mean translational and rotational displacements of all cases according to elapsed time (0-10 min in 2-min increments, 10-20 min in 5-min increments). Error bars represent standard errors. R-L: Right-left; S-I: superiorinferior; A-P: anterior-posterior.

$\mathrm{mm},>1^{\circ}$, or $>2^{\circ}$ according to the elapsed treatment time. RL displacements of $>1 \mathrm{~mm}$ and $>2 \mathrm{~mm}$ were observed at 4 6 and 8-10 minutes after treatment initiation, respectively. No displacement of $>2 \mathrm{~mm}$ was observed within $20 \mathrm{~min}$ in the S-I and A-P directions. Rotational displacements in the Yaw direction of $>1^{\circ}$ were observed at 10-15 min after treatment initiation.

\section{Discussion}

Some studies evaluated the intra-fractional spinal motion during SBRT using ExacTrac (BrainLab, Heimstetten, Germany) (8) or Cone-Beam CT (9). These studies analyzed the spinal motion from the positional data of pre- and postSBRT or those of very short period of time during SBRT. 
Table II. Proportion of cases with mean translational and rotational displacements of $>1 \mathrm{~mm},>2 \mathrm{~mm},>1^{\circ}$, or $>2^{\circ}$ according to elapsed treatment time. Parameters are given in percentages.

\begin{tabular}{|c|c|c|c|c|c|c|c|c|}
\hline & & $0-2 \mathrm{~min}$ & $2-4 \min$ & 4-6 min & $6-8 \mathrm{~min}$ & $8-10 \mathrm{~min}$ & $10-15 \min$ & $15-20 \mathrm{~min}$ \\
\hline \multirow[t]{2}{*}{ R-L } & $>1 \mathrm{~mm}$ & 0.0 & 0.0 & 12.5 & 12.5 & 21.9 & 31.3 & 40.6 \\
\hline & $>2 \mathrm{~mm}$ & 0.0 & 0.0 & 0.0 & 0.0 & 3.1 & 6.3 & 12.5 \\
\hline \multirow[t]{2}{*}{ S-I } & $>1 \mathrm{~mm}$ & 0.0 & 0.0 & 0.0 & 3.1 & 3.1 & 3.1 & 12.5 \\
\hline & $>2 \mathrm{~mm}$ & 0.0 & 0.0 & 0.0 & 0.0 & 0.0 & 0.0 & 0.0 \\
\hline \multirow[t]{2}{*}{ A-P } & $>1 \mathrm{~mm}$ & 0.0 & 0.0 & 0.0 & 0.0 & 0.0 & 0.0 & 9.4 \\
\hline & $>2 \mathrm{~mm}$ & 0.0 & 0.0 & 0.0 & 0.0 & 0.0 & 0.0 & 0.0 \\
\hline \multirow[t]{2}{*}{ Roll } & $>1^{\circ}$ & 0.0 & 0.0 & 0.0 & 0.0 & 0.0 & 0.0 & 0.0 \\
\hline & $>2^{\circ}$ & 0.0 & 0.0 & 0.0 & 0.0 & 0.0 & 0.0 & 0.0 \\
\hline \multirow[t]{2}{*}{ Pitch } & $>1^{\circ}$ & 0.0 & 0.0 & 0.0 & 0.0 & 0.0 & 0.0 & 0.0 \\
\hline & $>2^{\circ}$ & 0.0 & 0.0 & 0.0 & 0.0 & 0.0 & 0.0 & 0.0 \\
\hline \multirow[t]{2}{*}{ Yaw } & $>1^{\circ}$ & 0.0 & 0.0 & 0.0 & 0.0 & 0.0 & 3.1 & 3.1 \\
\hline & $>2^{\circ}$ & 0.0 & 0.0 & 0.0 & 0.0 & 0.0 & 0.0 & 0.0 \\
\hline
\end{tabular}

R-L: Right-left; S-I: superior-inferior; A-P: anterior-posterior.

Therefore, they did not evaluate the continuous spinal motion during the entire treatment. Murphy et al. (10) and Chuang et al. (11) have measured the intra-fractional motion with the CyberKnife X-ray imaging system, but only analyzed positional displacements between consecutive image acquisitions taken in 1-2 min intervals, rather than the positional displacement over the entire treatment fraction. Although intra-fractional motions of the skull or metal markers have been reported, there is no report on the timedependent intra-fractional spinal motion in SBRT (12).

Mean translational and rotational displacements were correlated with elapsed treatment time, indicating that the spine was systematically displaced during treatment and this displacement increased with time. Despite this, there was no case in which the maximum rotational displacement exceeded $1^{\circ}$ within $10 \mathrm{~min}$ from the initiation of the treatment. Moreover, the influence of rotational displacement is considered to be limited in spinal SBRT. The reason is that the beam isocenter is set near the center of the target in most situations (12). In contrast, translational displacements, especially in the R-L direction, were relatively large, with displacements of $>1 \mathrm{~mm}$ at 4-6 min and $>2 \mathrm{~mm}$ at 8-10 $\mathrm{min}$ from the initiation of the treatment in some cases. Hoogeman et al. have recommended patient position matching at least every 5 minutes in order to achieve irradiation accuracy in the millimeter or sub-millimeter range for skull or metal marker tracking (12). Similar findings were obtained in the present study, suggesting the importance of frequent image guidance for spinal SBRT due to the close proximity of organs at risk (OARs), especially the spinal cord.

In SBRT, steep dose distributions are applied to deliver a large dose to the tumor while reducing it to the adjacent OARs (4). Serious adverse events could occur if large doses are delivered to the OARs due to the intra-fractional motion. Therefore, when treating patients with an IGRT device that does not allow for real-time positional correction during irradiation, an appropriate setup margin must be set to account for intra-fractional motion (13). However, expanding the setup margin may cause overlap between the PTV and OARs, making it difficult to irradiate the tumor with a sufficiently large dose. In the present study, increasing intrafractional motion of the spine was observed as the treatment time elapsed. Therefore, it is required to either shorten the irradiation time or perform frequent image guidance.

This study has several limitations worth noting. First, the sample size was small, thus, we could not assess the influence of differences in tracking sites (cervical, thoracic, or lumbar spine). Depending on the fixation method, the cervical or upper thoracic spine may exhibit more positional displacements due to the curvature. Second, intra-fractional motion may change depending on the condition of the patient. When a patient has severe pain, for instance, the intra-fractional motion may be greater than the patients in the present study. Spinal SBRT sometimes requires the use of analgesics to maintain the patient's posture during treatment (14).

In summary, both time-dependent translational and rotational displacements were observed in SBRT for spinal metastasis. The results of the present study suggest that spine position may be checked at least every 4-6 min during SBRT or the treatment time should be limited to 4-6 min to ensure the irradiation accuracy within the millimeter or submillimeter range.

\section{Conflicts of Interest}

The Authors declare that there are no conflicts of interest.

\section{Authors' Contributions}

All Authors contributed to the study conception and design. Material preparation, data collection and analysis were performed by $\mathrm{RH}$ and 
YO. The first draft of the manuscript was written by RH and all authors commented on previous versions of the manuscript. All Authors read and approved the final manuscript.

\section{Acknowledgements}

This study was approved by the Institutional Review Board of Saitama Medical University International Medical Center (Authorization number: 18-106). This was a retrospective study, and thus the requirement for written informed consent was waived.

A part of this study was presented at the $20^{\text {th }}$ Asia-Oceania Congress on Medical Physics (AOCMP).

The Authors thank Jun Teranishi, Tomohito Nakada, Tohru Azami, and Haruka Yoshida (Division of Radiology, Saitama Medical University International Medical Center) for their assistance in data collection.

\section{References}

1 Kumar R, Nater A, Hashmi A, Myrehaug S, Lee Y, Ma L, Redmond K, Lo SS, Chang EL, Yee A, Fisher CG, Fehlings MG and Sahgal A: The era of stereotactic body radiotherapy for spinal metastases and the multidisciplinary management of complex cases. Neurooncol Pract 3(1): 48-58, 2016. PMID: 31579521. DOI: $10.1093 /$ nop/npv022

2 Zeng KL, Tseng CL, Soliman H, Weiss Y, Sahgal A and Myrehaug S: Stereotactic body radiotherapy (SBRT) for oligometastatic spine metastases: an overview. Front Oncol 9: 337, 2019. PMID: 31119099. DOI: 10.3389/fonc.2019.00337

3 Myrehaug S, Sahgal A, Hayashi M, Levivier M, Ma L, Martinez R, Paddick I, Régis J, Ryu S, Slotman B and De Salles A: Reirradiation spine stereotactic body radiation therapy for spinal metastases: systematic review. J Neurosurg Spine 27(4): 428435, 2017. PMID: 28708043. DOI: 10.3171/2017.2.SPINE16976

4 Benedict SH, Yenice KM, Followill D, Galvin JM, Hinson W, Kavanagh B, Keall P, Lovelock M, Meeks S, Papiez L, Purdie T, Sadagopan R, Schell MC, Salter B, Schlesinger DJ, Shiu AS, Solberg T, Song DY, Stieber V, Timmerman R, Tomé WA, Verellen D, Wang L and Yin FF: Stereotactic body radiation therapy: the report of AAPM Task Group 101. Med Phys 37(8): 4078-4101, 2010. PMID: 20879569. DOI: 10.1118/1.3438081

5 Alaei P and Ding GX: Overview of image guidance in radiation therapy. In: Alaei P, Ding GX (eds). Image guidance in radiation therapy: Techniques, accuracy, and limitations. $1^{\text {st }}$ ed. Tennessee: American Association of Physicists in Medicine by Medical Physics Publishing, pp. 1-22, 2018.

6 Gallo JJ, Kaufman I, Powell R, Pandya S, Somnay A, Bossenberger T, Ramirez E, Reynolds R, Solberg T and Burmeister J: Single-fraction spine SBRT end-to-end testing on TomoTherapy, Vero, TrueBeam, and CyberKnife treatment platforms using a novel anthropomorphic phantom. J Appl Clin Med Phys 16(1): 5120, 2015. PMID: 25679169. DOI: 10.1120/ jacmp.v16i1.5120
7 Yang J, Ma L, Wang XS, Xu WX, Cong XH, Xu SP, Ju ZJ, Du L, Cai BN and Yang J: Dosimetric evaluation of 4 different treatment modalities for curative-intent stereotactic body radiation therapy for isolated thoracic spinal metastases. Med Dosim 41(2): 105-112, 2016. PMID: 26831753. DOI: 10.1016/ j.meddos.2015.10.003

8 Jin JY, Ryu S, Rock J, Faber K, Chen Q, Ajlouni M and Movsas $\mathrm{B}$ : Evaluation of residual patient position variation for spinal radiosurgery using the Novalis image guided system. Med Phys 35(3): 1087-1093, 2008. PMID: 18404943. DOI: 10.1118/ 1.2839097

9 Li W, Sahgal A, Foote M, Millar BA, Jaffray DA and Letourneau D: Impact of immobilization on intrafraction motion for spine stereotactic body radiotherapy using cone beam computed tomography. Int J Radiat Oncol Biol Phys 84(2): 520526, 2012. PMID: 22401920. DOI: 10.1016/j.ijrobp.2011.12.039

10 Murphy MJ, Chang SD, Gibbs IC, Le QT, Hai J, Kim D, Martin DP and Adler JR Jr: Patterns of patient movement during frameless image-guided radiosurgery. Int J Radiat Oncol Biol Phys 55(5): 1400-1408, 2003. PMID: 12654453. DOI: 10.1016/ s0360-3016(02)04597-2

11 Chuang C, Sahgal A, Lee L, Larson D, Huang K, Petti P, Verhey $\mathrm{L}$ and Ma L: Effects of residual target motion for image-tracked spine radiosurgery. Med Phys 34(11): 4484-4490, 2007. PMID: 18072513. DOI: $10.1118 / 1.2790587$

12 Hoogeman MS, Nuyttens JJ, Levendag PC and Heijmen BJ: Time dependence of intrafraction patient motion assessed by repeat stereoscopic imaging. Int J Radiat Oncol Biol Phys 70(2): 609-618, 2008. PMID: 17996389. DOI: 10.1016/j.ijrobp. 2007.08.066

13 Landberg T, Chavaudra J, Dobbs J, Gerard JP, Hanks G, Horiot JC, Johansson KA, Möller T, Purdy J, Suntharalingam N and Svensson H: International Commission on Radiation Units and Measurements (ICRU) report 62, prescribing, recording and reporting photon beam therapy (Supplement to ICRU Report 50), Bethesda: ICRU Publications, pp. 1-52, 1999.

14 Yang $M$ and Timmerman R: Stereotactic ablative radiotherapy uncertainties: delineation, setup and motion. Semin Radiat Oncol 28(3): 207-217, 2018. PMID: 29933881. DOI: 10.1016/ j.semradonc.2018.02.006
Received March 18, 2021

Revised April 16, 2021

Accepted April 20, 2021 